\title{
Intoxication With Bourbon Versus Vodka: Effects on Hangover, Sleep, and Next-Day Neurocognitive Performance in Young Adults
}

\author{
Damaris J. Rohsenow, Jonathan Howland, J. Todd Arnedt, Alissa B. Almeida, Jacey \\ Greece, Sara Minsky, Carrie S. Kempler, and Suzanne Sales
}

\begin{abstract}
Background: This study assessed the effects of heavy drinking with high or low congener beverages on next-day neurocognitive performance, and the extent to which these effects were mediated by alcohol-related sleep disturbance or alcoholic beverage congeners, and correlated with the intensity of hangover.

Methods: Healthy heavy drinkers age 21 to $33(n=95)$ participated in 2 drinking nights after an acclimatization night. They drank to a mean of $0.11 \mathrm{~g} \%$ breath alcohol concentration on vodka or bourbon one night with matched placebo the other night, randomized for type and order. Polysomnography recordings were made overnight; self-report and neurocognitive measures were assessed the next morning.

Results: After alcohol, people had more hangover and more decrements in tests requiring both sustained attention and speed. Hangover correlated with poorer performance on these measures. Alcohol decreased sleep efficiency and rapid eye movement sleep, and increased wake time and next-day sleepiness. Alcohol effects on sleep correlated with hangover but did not mediate the effects on performance. No effect of beverage congeners was found except on hangover severity, with people feeling worse after bourbon. Virtually no sex differences appeared.

Conclusions: As drinking to this level affects complex cognitive abilities, safety could be affected, with implications for driving and for safety-sensitive occupations. Congener content affects only how people feel the next day so does not increase risk. The sleep disrupting effects of alcohol did not account for the impaired performance so other mechanisms of effect need to be sought. As hangover symptoms correlate with impaired performance, these might be contributing to the impairment.
\end{abstract}

Key Words: Hangover, Polysomnography, Neuropsychology, Alcohol Administration, Congeners, Residual Alcohol Effects.

E VIDENCE IS MIXED on whether the residual effects of heavy drinking cause performance deficits the day after intoxication. "Residual effects" refers to any subjective, physiological, and/or behavioral effects of heavy drinking when blood alcohol concentration (BAC) has fallen to near zero after an episode of heavy drinking. Hangover is the subset of residual effects defined by symptoms, typically headache, nausea, thirst, and fatigue, that peak when BAC reaches $0 \mathrm{~g} \%$ (Rohsenow et al., 2007). Residual alcohol effects are of importance to the extent that they may affect safety-sensitive

From the Center for Alcohol and Addiction Studies (DJR, SS), Brown University, Providence, Rhode Island; Youth Alcohol Prevention Center at Boston University, School of Public Health (JH, ABA, $J G, \quad S M, C S K)$, Boston, Massachusetts; University of Michigan Medical School (JTA), Ann Arbor, Michigan.

Received for publication June 8, 2009; accepted October 13, 2009.

Reprint requests: Damaris J. Rohsenow, PhD, Center for Alcohol and Addiction Studies, Brown University, Box G-S121-5, Providence, RI 02912; Fax: 401-863-6697; E-mail: Damaris_Rohsenow@brown. $e d u$

Copyright (C) 2009 by the Research Society on Alcoholism.

DOI: $10.1111 / \mathrm{j} .1530-0277.2009 .01116 . x$ occupational performance, driving, or student learning or performance (Howland et al., 2006).

Surveys and qualitative studies find positive relationships between frequency of intoxication or hangovers and the frequency of workplace performance problems (Ames et al., 1997; Mangione et al., 1999), and of poorer college grades (Singleton and Wolfson, 2009). Experimental studies show residual effects of heavy drinking on occupational performance the next morning using workplace simulators (Chait and Perry, 1994; Finnigan et al., 1998; Lemon et al., 1993; Morrow et al., 1990, 1991, 1993; Streufert et al., 1995; Taylor et al., 1994, 1996; Törnros and Laurell, 1991; Wolkenberg et al., 1975; Yesavage et al., 1994; Yesavage and Leirer, 1986). Using neuocognitive tasks specifically, while negative results were found for some measures (Finnigan et al., 1998; Lemon et al., 1993; McCaul et al., 1991; Verster et al., 2003), documented detrimental effects were found for visual perception (Dowd et al., 1973); codification and identification tasks (Myrsten et al., 1980); alertness (Roehrs et al., 1991); divided attention and tracking (Roehrs and Roth, 2001a,b); eye-hand and multi-limb coordination, and attention (Seppälä et al., 1976); immediate and delayed (1 hour) free recall (Verster 
et al., 2003); visual, memory, and intellectual processing (Kim et al., 2003; McKinney and Coyle, 2004); time-reaction error in a go-no-go task (Alford and Wadling, 2004); sustained attention/reaction time (Finnigan et al., 2005); and choice reaction time (Kruisselbrink et al., 2006; McKinney and Coyle, 2004; Seppälä et al., 1976). Inconsistencies among study findings may be the result of factors such as the type of performance measured, the amount of alcohol administered, age and experience of participants, length of time from drinking to testing, and confounds. When some studies tested residual effects before BAC returned to near zero, alcohol effects confounded the residual effects.

Mechanisms accounting for the residual effects of alcohol on performance have received less investigation, despite a number of hypotheses (Swift and Davidson, 1998; Wiese et al., 2000). Some of the possible mediators considered below include sleep disturbance effects of intoxication (Rohsenow et al., 2006), effects of beverage congeners (Nathan et al., 1970), and distracting effects of unpleasant hangover symptoms.

Consuming alcohol ( 0.16 to $1.0 \mathrm{~g} / \mathrm{kg}$ ) before bed produces reliable changes in sleep continuity and sleep architecture in young, healthy adults. Alcohol initially reduces sleep onset latency, and may increase total sleep time at low doses $(0.16 \mathrm{~g} / \mathrm{kg})$ but not at moderate or high doses (Stone, 1980). Most studies with low to moderate doses report no significant changes in sleep efficiency (percent of time devoted to sleep actually asleep) (Roehrs et al., 1991). Light Stage 1 sleep has been found to both increase (Kobayashi et al., 1998; Williams et al., 1983) and decrease (Roehrs et al., 1991) following alcohol ingestion. Sleep effects can differ in the first half of the night, when alcohol is being metabolized, from the second half, when it is being eliminated. The first half of the night typically has enhanced slow wave sleep and reduced rapid eye movement (REM) sleep (less time in REM, longer latency to REM) (Gillin et al., 2005; Kobayashi et al., 1998; MacLean and Cairns, 1982; Roehrs et al., 1991; Williams et al., 1983; Yules et al., 1966, 1967). The second half of the night is characterized by increased wakefulness and light Stage 1 sleep (Gillin et al., 2005; Knowles et al., 1968; MacLean and Cairns, 1982; Roehrs and Roth, 2001a,b; Rundell et al., 1972; Williams et al., 1983) and a rebound in REM (Roehrs and Roth, 2001a,b). Thus, sleep is lighter and more disturbed, particularly during the second half of the night. Subjectively, young adults reported improved sleep quality after drinking beer to $0.11 \mathrm{~g} \%$ BAC versus placebo; with their perception possibly influenced by the more rapid sleep onset they reported (Rohsenow et al., 2006). However, the objectively poorer sleep particularly in the second half of the night could result in impaired performance after awakening.

Most alcoholic beverages contain small amounts of chemicals other than ethanol as a by-product of the materials used in the fermenting process (e.g., grains and wood casks). Congeners are complex organic molecules with toxic effects including acetone, acetaldehyde, fusel oil, tannins, and furfural, with bourbon having 37 times the amount of congeners as vodka (Nathan et al., 1970). While methanol has also been implicated (Calder, 1997), the elimination of methanol from the body coincides with the onset of hangover, although it does leave formaldehyde and formic acid as byproducts (Jones, 1987). In some studies (Katkin et al., 1970), but not others (McMurphree et al., 1966; Nathan et al., 1970), intoxication with bourbon was more impairing than with vodka. However, high-congener beverages such as bourbon did produce more hangover than low congener beverages (Chapman, 1970) and more electroencephalogram (EEG) signs of drowsiness (McMurphree et al., 1966). While the main cause of hangover symptoms is ethanol (e.g., Chapman, 1970; Ylikahri et al., 1974), congeners may increase symptom severity. However, this aspect has been virtually unstudied as these early studies and never examined for effects on performance measures.

Finally, the experience of headache, nausea, and other physical discomfort might interfere with attention, concentration, or rapid responses, abilities central to many safety-sensitive operations. While symptoms cannot be experimentally manipulated separately from other residual effects, correlational data can be supportive or disconfirming. The only study exploring this hypothesis found no association between nextday psychomotor skill impairment and intensity of hangover (Seppälä et al., 1976).

This study investigated the effects of drinking to above legal intoxication on next-day neurocognitive performance and the extent to which these effects were mediated by alcohol-related sleep disturbance or alcoholic beverage congeners, and correlated with the incidence and intensity of hangover. Sleep was assessed using polysomnography and neurocognitive performance was measured using selected neurobehavioral tasks. While Prat and colleagues (2008) recommended using neuropsychological tests that have shown results across various drug effects (executive or frontal function, impulsive decision making), hangover involves the absence rather than presence of drug. As the primary symptoms expected to affect neurocognitive performance are fatigue, dysphoria, and difficulty concentrating, more specific hypotheses were made based on these effects so as to reduce the number of tests to the most relevant ones. The hypotheses were that heavy drinking would result in decrements in abilities requiring speed or sustained concentration (e.g., sustained attention/vigilance and reaction time), and more disrupted lighter sleep (decreased sleep efficiency because of more time awake and more wake-ups, less time in REM sleep, and increased light Stage 1 sleep), and that the sleep deficits would mediate the effects of alcohol on the performance measures. Hypotheses about congener content were that the high-congener beverage, compared to the low congener beverage, would result in more hangover, decrements in neurocognitive performance requiring speed or sustained concentration, and more disrupted sleep. Finally, degree of hangover was hypothesized to correlate with the sleep decrements and with the degree of performance decrements after heavy drinking. 


\section{MATERIALS AND METHODS}

\section{Participants}

Participants were university students or recent graduates recruited by advertisements from greater Boston, Massachusetts. They needed to be (i) between 21 and 35 years of age (actual range $=21$ to 33); (ii) score $<5$ on the Short Michigan Alcohol Screening Test (Selzer et al., 1975) and no history of treatment or counseling for alcohol problems; (iii) $\geq 5$ drinks on a single occasion ( $\geq 4$ if female) at least once in the 30 days prior to screening; (iv) no health problems or current medication use contraindicated for alcohol; (v) no sleep disorders; (vi) graduated from or currently attending a college or university; and (vii) negative pregnancy test and not nursing, if female. Females were not scheduled by menstrual cycle (Brick et al., 1986; Niaura et al., 1987; Terner and deWit, 2006). Participants were required to abstain from alcohol, illicit drugs, sleep aids, and caffeine for 24 hours prior and food/beverages for 3 hours prior to their evening sessions. Participant characteristics are shown in Table 1. There were no significant differences in background characteristics by beverage type administered. Genders did not differ significantly in quantity or frequency of typical drinking.

Participants were paid \$450. The Institutional Review Boards at Boston Medical Center, Brown University, and the University of Michigan approved this study.

\section{Study Design}

The design was $2 \times 2 \times 2$ : alcohol content (alcohol vs. placebo) by congener (high vs. low congener alcohol) by order (alcohol on Day 1 vs. alcohol on Day 2). Alcohol content was the within subjects factor; congener and order were between-groups factors. The high-congener beverage was bourbon, the low congener beverage vodka.

\section{Procedures}

Screening. Individuals responding to advertisements were screened for exclusionary criteria and examined by a physician after informed consent. To ensure stable sleep without sleep deprivation, they were instructed to maintain an 8 hours time-in-bed schedule (starting no later than midnight) and avoid napping for 3 days before testing, confirmed with a sleep diary, activity monitor (Octagonal Basic Motionlogger; Ambulatory Monitoring, Inc., Ardsley, NY), and evening and morning calls to a time-stamped answering machine. Before beverage administration, participants who reported consuming alcohol, caffeine, prescription or overthe-counter drugs not approved by the study physician or napping within the prior 24 hours, or food or beverage within the prior 3 hours, were rescheduled; those presenting with a positive breath alcohol concentration $(\mathrm{BrAC})$ were excluded from further participation.

Table 1. Participant Characteristics

\begin{tabular}{lc}
\hline & $M(\mathrm{SD})$ or $n(\%)$ \\
\hline Male (\%) & $37(39 \%)$ \\
White & $79(83 \%)$ \\
Black & $3(3 \%)$ \\
Asian & $4(4 \%)$ \\
Other race & $9(10 \%)$ \\
In college currently & $29(31 \%)$ \\
Age & $24.5(2.8)$ \\
No. drinks on typical day & $3.4(1.5)$ \\
Drank at least once per week & $87(92 \%)$ \\
High or drunk $\geq 3$ days/month & $62(65 \%)$ \\
\hline
\end{tabular}

Note: Total $n=95$.
Session Procedures and Randomization. Groups of 3 to 4 participants returned for 3 overnight sessions, the first for sleep screening and acclimatization to polysomnography equipment, and second and third for experimental drinking sessions (see Table 2). Some had alcohol and some placebo on each drinking night. Participants were told they would receive alcohol one night and placebo the other night, with a 50-50 chance of receiving alcohol the first night and a 50-50 chance of having vodka versus bourbon.

First overnight session: beginning at 7:00 PM, participants practiced neurocognitive tests. At 10:00 PM, polysomnography electrodes were applied. At 11:00 PM participants had lights out in individual sleeping quarters, continuously monitored by a polysomnographic technologist and medical technician overnight (electrode units allow trips to the attached toilet room). At 7:00 AM participants were awakened, their electrodes were removed, they completed a baseline hangover severity and symptom scale, and they received breakfast (no coffee). From 8:00 to 9:30 AM, they performed the neurocognitive tests (data not used).

The following day, participants reported at 4:00 PM for the first experimental session. Following screening for compliance with requirements, participants received a standardized meal, then were randomized to beverage type (bourbon or vodka) and order (alcohol on first or second night).Following the beverage administration from 8:30 to 10:00 PM, polysomnography electrodes were applied while additional $\mathrm{BrAC}$ tests were conducted periodically. After a 15 minute absorption period, subjective ratings of abilities were completed. At 11 PM, participants had lights out for an 8-hour opportunity to sleep.

Participants were awakened at 7:00 AM, completed the hangover measure, ate breakfast (no coffee), and were breath-tested. From 8:00 to 9:30 Am they completed neurocognitive tests and some ratings (start time delayed if BrAC $>0.01$ until below that), allowing an hour after waking to avoid confounding by sleep inertia (Tassi and Muzet, 2000). One week later they returned for the second experimental session, identical except for beverage received.

\section{Beverage Administration Procedures}

The alcoholic beverages were bourbon (101 proof Wild Turkey®, Austin, Nichols Distilling Co., Lawrenceburg, KY) or vodka (100 proof Absolut ${ }^{\circ}, \mathrm{V} \& S$ Vin \& Sprit AB, Stockholm, Sweden) mixed with chilled caffeine-free cola (Coke ${ }$, The Coca-Cola Co., Atlanta, GA) in a 1:4 ratio (Rohsenow and Marlatt, 1981). The cola was designed to mask the color differences and partially mask the taste.

Table 2. Timeline of Study Procedures During Each Session

\begin{tabular}{|c|c|}
\hline essions & \\
\hline Evening & \\
\hline $4: 00$ to $4: 15$ PM & Screened for adherence to study protocol. \\
\hline $4: 15$ to $5: 00$ PM & Dinner. Weighed. \\
\hline $6: 00$ to $8: 00 \mathrm{PM}$ & Polysomnography leads placed. \\
\hline $8: 30$ to $10: 00 \mathrm{PM}$ & Beverage consumption. \\
\hline $10: 00$ to $10: 30 \mathrm{PM}$ & Evening ratings, then snacks. \\
\hline $10: 15$ PM & BrAC test, extra drink if low BrAC. \\
\hline $10: 30$ to $11: 00$ PM & Prepare for bed. BrAC tests every 15 minutes \\
\hline $11: 00 \mathrm{pM}$ to $7: 00 \mathrm{Am}$ & $\begin{array}{l}\text { Lights out, polysomnography monitoring. } \\
\text { Observed by medical and sleep technicians. }\end{array}$ \\
\hline Morning & \\
\hline $7: 00$ to $7: 30 \mathrm{AM}$ & $\begin{array}{l}\text { Awaken, BrAC test, hangover scale, } \\
\text { morning ratings. }\end{array}$ \\
\hline $7: 30$ to $8: 00 \mathrm{AM}$ & $\begin{array}{l}\text { Breakfast, then BrAC test } \\
\text { (if previous one positive). }\end{array}$ \\
\hline $8: 00$ to $9: 15 \mathrm{AM}$ & $\begin{array}{l}\text { Neuropsychological tests } \\
\text { (delayed if BrAC > } 0.01 \mathrm{~g} \% \text { ). }\end{array}$ \\
\hline $9: 15$ to $10: 00 \mathrm{AM}$ & Post-test ratings, pay (last day). \\
\hline
\end{tabular}

BrAC, breath alcohol concentration. 
The placebo for both of these beverages was chilled caffeine-free cola plus de-carbonated tonic (for a bitter taste), in amounts equivalent to the alcoholic beverage, with a few drops of vodka or bourbon floated on top. A BrAC of $0.10 \mathrm{~g} \%$ was targeted based on dose-response studies (e.g., Chapman, 1970) which indicated that obtaining a BrAC of at least $0.10 \mathrm{~g} \%$ was a better determinant of hangover symptom severity than a $\mathrm{g} / \mathrm{kg}$ dosage of alcohol per se. This required $1.2 \mathrm{~g} / \mathrm{kg}$ body weight for men and $1.1 \mathrm{~g} / \mathrm{kg}$ for women (Friel et al.,1999), with redosing as described below for individuals who did not reach the target BrAC.

Between 8:30 PM and 10:00 PM beverages were consumed, using pacing procedures. Participants were told not to inspect each others' drinks nor discuss thoughts about the beverage received. Research assistants who interacted with participants and collected measures other than $\mathrm{BrAC}$ were blind to beverage condition. The beverage preparation assistant had no other contact with participants except to collect $\mathrm{BrAC}$ readings. If participants randomized to alcohol did not reach $0.10 \mathrm{~g} \% \mathrm{BrAC}$ when tested 15 minutes after finishing the beverage, the ratio of obtained versus target $\mathrm{BrAC}$ was used to estimate an additional amount of the same beverage to consume within 5 minutes. To maintain blinding, one or more participants who drank placebo were given a matched extra dose when logistics allowed. BrACs were tested every 15 minutes until 11:00 PM. After achieving their target BrAC, participants completed subjective measures, received snacks and were escorted to their bedrooms for lights out at 11:00 PM.

\section{Individual Difference Measures}

Recent drinking practices (past month) were assessed with a 3-item alcohol use questionnaire: 1) "Considering all your drinking times in the past 30 days, about how often did you have any beer, wine or liquor?," Likert-rated from 1 "once a day" to 7 "did not drink" with each point anchored. 2) "In the past 30 days, on a typical day that you drank, about how much did you have to drink in one day?," rated from 1 to 8 , with choices of 1 to 7 drinks and " 8 or more drinks," followed by a question asking how many drinks for those answering the last item. One drink was defined as $12 \mathrm{oz}$ of beer or wine cooler, $4 \mathrm{oz}$ of wine or $1 \mathrm{oz}$ of liquor. Average daily volume of alcohol intake was the product of these. 3) They rated how often they got "high or drunk or "had a buzz on"" after drinking in the past 30 days rated from 1 "once a day" to 8 "never."

\section{Dependent Measures of Residual Alcohol Effects}

Hangover. The Acute Hangover Scale (AHS; Rohsenow et al., 2007), developed based on empirical hangover data (Chapman, 1970; Roehrs et al., 1991; Ylikahri et al., 1974), consists of 8 symptoms validated empirically plus "hangover" (in lieu of the valid but out-of-use term "malaise") rated from 0 "none" to 7 "incapacitating" on anchored Likert-type scales. (Other physical signs reported from surveys were not supported in laboratory studies.) The 9 items form a reliable and valid scale, scored using the mean.

Neuropsychological Tests. Tests selected were those most apt to be affected by the residual effects of heavy alcohol consumption because of requiring speed or sustained attention/concentration. To minimize overlap with similar metrics, when tests were done using each hand, only the test with preferred hand was chosen as more relevant, and when tests had forward and backward versions, the backward version was chosen as more difficult and thus more likely to be sensitive in a population without brain damage. Participants practiced the tests under both evening and morning conditions to reduce learning effects.

Psychomotor Vigilance Task. The PVT (Dinges and Powell, 1985 ) tests both visual sustained attention and reaction time (Ambulatory Monitoring, Inc.). For 5 minutes, participants have to press a button on the hand-held unit as quickly as possible in response to numbers scrolling on the LCD screen with a 3 to 7 seconds interstimulus interval. The primary variable is median reaction time in milliseconds.

Nine tests were selected from the Neurobehavioral Evaluation System 3 (White et al., 2003) assessing neurocognitive functioning. The computer provided verbal instructions (via headphones) and responses were made using the touch screen monitor. One test assesses sustained and selective attention as well as reaction time: Continuous Performance Test (CPT), scored for reaction time in milliseconds. The following tests assessing speed or reaction time were selected: Finger Tapping Test, preferred hand (FTT-P) (assesses manual motor speed and dexterity); Sequence Test A latency; DigitSymbol Test latency; Pattern Memory Test latency (all assessing speed of cognitive processing). The following tests assessing sustained attention were selected: Auditory Digit Span Test, Backward; Adaptive Paced Auditory Serial Addition Test, number correct; Visual Span Test, backward; and Pattern Memory Test, number correct.

\section{Dependent Measures of Sleep and Next-Day Sleepiness}

Polysomnography Variables. The 5 most relevant sleep measures used to test the hypotheses included: sleep efficiency (total sleep time divided by lights out time), wake time after sleep onset (minutes awake from sleep onset to "lights on"); number of nighttime awakenings (number of EEG-defined arousals during total sleep period); and the percent of total sleep time that was in REM (REM\%) or Stage 1 Sleep. Variables were not scored by first versus second half of night for this study both to minimize the number of variables used in hypothesis testing and because hypothesized effects on next-day performance should be based on disruption evident in total scores (i.e., not disrupted in one half but improved in the other half). Variables not used in hypothesis testing included the following: Latency to REM was not used because it is conceptually redundant with REM \% while being less relevant to effects on next-day performance. Total sleep time (minutes between sleep onset and the last epoch of sleep minus time awake between these) was not used in hypotheses because it was collinear with $(r=0.93)$ while less relevant than sleep efficiency. Sleep onset latency (minutes from "lights out" to the first of 3 consecutive 30 second epochs of any stage of sleep), and percent time in and latency to Stage 2 Sleep or Slow Wave Sleep (Stages 3 and 4 sleep) were not used because they were not hypothesized to be disrupted. For informational purposes, a few sleep variables sometimes improved by intoxication were also analyzed (selecting only ones not collinear with others): Sleep onset latency, total sleep time, and percent time in Stage 1 or 2 sleep.

Sleep Quality Ratings. From a subjective measure of sleep quality (Roehrs et al., 1991), we used 4 fully anchored Likert ratings of sleep quality, sleep refreshment, level of alertness, and ability to concentrate, the first 2 rated from "much better" (1) to "much worse" (5) (reverse scored in calculations), and the other 2 rated from "extremely poor" (1) to "excellent" (7). The mean of the 4 refreshment/ quality items is used because they form a reliable and valid scale (Roehrs et al., 1991; Rohsenow et al., 2006); the latency and length items are used individually. This measure is to provide information about perceived effects of alcohol and/or congeners on perceived sleep quality, as people's perceptions determine their behavioral choices.

Subjective Sleepiness. One element of hangover is fatigue and congeners caused signs of drowsiness (McMurphree et al., 1966). Sleepiness on arising was assessed using the Stanford Sleepiness Scale (SSS; Hoddes et al., 1973). This involves endorsing 1 of 7 descriptors that form a single Likert scale from 1 "Feeling active and vital; wide awake" to 7 "Almost in reveries; sleep onset soon; lost struggle to stay awake." 


\section{Subjective Performance Measures}

As people have no past experience with the cognitive tests against which to compare their performance, rating their driving ability would give a better metric of how impaired they perceived themselves to be. To assess participants' perceptions of their impairment in performance, participants rated 5 items on ability to drive a vehicle after drinking in the evening: "Right now, would your ability to operate a vehicle be better or worse than usual?" and "What you drank here has made your ability to operate a vehicle right now" were rated on 5 -point fully anchored scales from 1 "much worse" to 5 "much better." "How likely is it that you would operate a vehicle the way you feel right now?" was rated on a 5-point fully anchored scale from 1 "definitely would not" to 5 "definitely would." Two more items asked how well they thought they would drive a vehicle right now and as compared to how they usually drive. These items were not used because of conceptual overlap with the above items and because they were not repeated in the morning. In the morning, participants again rated the first 3 items.

\section{Alcohol Administration Manipulation Checks}

Participant beverage beliefs were checked by asking whether they believed they had received vodka, bourbon, or placebo with the caffeine-free cola. They were also asked "How intoxicated do you feel right now?" rated "not at all" (1), "mildly" (2), "moderately" (3), "very" (4), or "completely" (5). Breath alcohol was assessed using an AlcoSensor-4 (Intoximeters, Inc., St Louis, MO).

\section{Data Analysis}

All variables were normally distributed except where indicated; those were transformed as indicated to correct skewness. All analyses were carried out in SPSS-PC (SPSS Inc., Chicago, IL). The 4 items in the sleep quality scale were checked for reliability using Cronbach's $\alpha$. The AHS was not rechecked for reliability because these AHS data had been combined with 2 other data sets and the combined reliability was reported in Rohsenow and colleagues (2007). Alpha needs to be determined based on hypothesis-wide number of analyses (per Dar et al., 1994). While sex and order were checked (exploratory), these do not test hypotheses, and analyses of alcohol by congener content (hypotheses) inherently include corrections for including the 2 main effects and the interaction term within each analysis. Therefore, rather than number of tests, the number of variables used in these tests for (i) tests of acute affects, (ii) tests of residual effects, (iii) tests of relationships of hangover to impaired performance, and (iv) tests of the interaction term in the mediation analyses are considered separately. Multivariate analyses were not used because we were not interested in the linear combination of the variables. Given the hypothesis-wide number of dependent variables and as Bonferroni's corrections are known to overcorrect, the probability level of $\alpha=0.005$ was selected for the tests of residual effects, 0.01 for sleep effects ( 5 objective and 2 subjective measures) and mediation, and 0.05 for acute effects and for correlations. Power analyses indicated that tests of about $p<0.02$ or better were needed to detect medium effect sizes.

Repeated measures analyses of variance (SPSS's MANOVA procedure) were conducted for each continuous dependent variable first as $2 \times 2$ alcohol content by order MANOVAs to rule out order effects in alcohol results first, then as $2 \times 2$ alcohol content by gender MANOVAs to rule out interaction with sex, and finally as $2 \times 2$ alcohol content by congener content analyses to test the hypotheses that alcohol effects differed by congener content of beverages. While main effects for alcohol were reported, only interaction effects for order, gender or congeners were of interest, not main effects or higher-order interactions. When the interpretation of interaction tests was not obvious on inspection, simple effects tests within the MANOVA procedure were used to compare alcohol versus placebo values within each level of gender or of order. Main effects for gender were inspected but did not pertain to any hypotheses, given genderadjusted dosing. A $2 \times 2 \times 2 \times 2$ analysis was not run because cell sizes would have been too small for adequate power and high-order interactions would have been very difficult to interpret.

The relationships of hangover (AHS score) to neuropsychological measures and to polysomnography measures that were affected by alcohol were investigated using correlations within the alcohol condition only. Mediational analyses could not be done using the hangover measure because it has essentially no variance in the placebo condition. Also, as hangover occurs at the same time as performance decrements, mediational causality could not be inferred.

Mediational analyses of residual effects on neuropsychological performance measure by alcohol-induced decrements in sleep were conducted using the 2 objective measures that were found to be most strongly disrupted by alcohol, as they satisfy part of the requirements of mediation. Measures of performance found impaired by alcohol were used as the dependent variables. Mediational effects were analyzed using the test of joint significance method of MacKinnon and colleagues (2002): both the path between alcohol condition and the sleep mediator, and the path from the sleep mediator to the performance outcome need to be significant to support mediation (the bootstrapping estimation approach was not needed because of adequate sample size). The first 2 of these conditions is conceptually redundant with the analyses of main effects, so it is the other condition that needs to be met for mediation. Because the 2 variables were repeated on an alcohol day and a placebo day, the mean of those values across the 2 conditions was entered into each linear regression of performance measure on sleep measure. Four regressions were conducted, using the 2 significantly affected sleep variables and the 2 significantly affected performance measures.

Statistical effect sizes for analyses of variance using continuous measures were reported using Cohen's (1988) $f$, as recommended for such analyses, where $f$ from 0.25 to 0.39 is a medium effect, smaller numbers are small effects, and $f \geq 0.40$ is a large effect. For regressions, effect sizes are the semi-partial squares $\left(s r^{2}\right.$; the change in $R^{2}$ because of the term) as these indicate the amount of variance accounted for. According to Cohen (1988), a $s r^{2}$ of 0.13 to 0.26 is medium, with lower values small and higher values large. Pearson's correlations of 0.30 to 0.40 are medium and $<0.30$ are small effects.

\section{RESULTS}

\section{Participant Enrollment}

Of 122 participants enrolled, 22 failed to complete the study and another 5 failed to reach the minimum BrAC of $0.09 \mathrm{~g} \%$. Of the remaining 95 participants, 10 did not have complete polysomnography data on both nights because of equipment malfunction (9 on placebo and 7 on alcohol). Because of occasional other missing values, 89 to 95 participants are included in the analyses of primary neurocognitive and self-report outcomes and 85 are included in analyses of sleep effects.

\section{Alcohol Administration Manipulation Checks}

Peak BrAC was $M=0.11( \pm 0.01 \mathrm{SD}) \mathrm{g} \%$, range from 0.09 to $0.15 \mathrm{~g} \%$. In the alcohol conditions, 20 people required more and 7 required less beverage than the formula indicated the first night, and 25 people required more and 6 required less beverage the second night than the formula indicated to obtain the target BrAC. Subjective intoxication 
ratings were higher after alcohol $(M=3.26 \pm 0.92)$ than placebo $(M=1.20 \pm 0.54)$ with a large statistical effect $(f=1.95), \quad F(1,92)=340.72, p<0.001$. No significant effects of order, gender or congener type were found. When alcohol was administered, the vodka versus bourbon deception worked well for the 52 people who received vodka (20 said they got vodka, 30 said bourbon, 1 said placebo, 1 did not answer) but not for the 43 people receiving bourbon (36 said they got bourbon, 4 vodka, 3 placebo). When placebo was consumed, $84 \%$ of participants $(n=80)$ said they received placebo, 10 said they received bourbon, and 3 said they received vodka. Beverage belief reports did not differ by gender or congener type in chi square tests.

\section{Measures of Residual Alcohol Effects}

See Table 3 for results for all self-report measures and Table 4 for significant results for performance measures (neuropsychological and sleep).

Hangover. The main effect for alcohol and the interaction of alcohol with congener content were both significant, with a large effect size for alcohol content and a medium effect size for the interaction. Hangover ratings were higher after alcohol than placebo, and higher in the alcohol condition after bourbon than vodka (note: 2 persons in the placebo condition reported a "mild" hangover). No significant effects of order or sex were seen.

Neuropsychological Tests. The 2 tests requiring both sustained attention and reaction time were significantly affected by alcohol 9 to 10 hours after the end of drinking, with no significant effects of order, gender or congeners. CPT reaction time was significantly $(p<0.002)$ impaired the morning after alcohol, with a medium effect size. People were $2 \%$ slower after alcohol than placebo, with no effects of order, gender, or congeners. With the PVT, the main effect for poorer results after alcohol was significant with a large effect size. One test requiring only speed, FTT-P, tended to be affected by alcohol $(p<0.04)$, accounted for by a significant interaction with order. When alcohol was given on the second of the 2 nights, the difference in number of taps between conditions was greater $(M=2.2)$ than when alcohol was given first $(M=0.6)$. The other 7 neuropsychological tests were not significantly affected by alcohol, order, sex, or congener content.

\section{Effects on Sleep}

Polysomnography Effects. Total sleep time and sleep efficiency were both negatively skewed so the square root of the reflected value was used in analyses; the untransformed value is displayed to ease interpretation. For sleep onset latency and wake time after sleep onset, 1 outlier was recoded into 1 plus the next highest value, per Tabachnick and Fidell (1996). Without alcohol, amount of time awake was collinear with sleep efficiency $(r=0.91)$ and total sleep time $(r=0.85)$. No other pairs of sleep variables were collinear except sleep efficiency with total sleep time $(r=0.93)$.

Alcohol condition showed a main effect for 3 of the 5 hypothesized sleep variables: sleep efficiency and REM $\%$ decreased while amount of wake time after sleep onset increased (see Table 4) after alcohol relative to placebo. For sleep efficiency there was also an interaction with gender, $F(1,83)=5.49, p<0.011$, with a medium effects size of $f=0.29$ : Men did not spend more time awake after alcohol $(M=31.9 \pm 21.3$ minutes $)$ than placebo $(M=32.6 \pm$ 24.9 minutes), but women spent more time awake after alcohol $(M=37.5 \pm 22.3$ minutes $)$ than placebo $(M=24.2 \pm$ 18.5 minutes). For number of awakenings after sleep onset, there was a significant interaction effect of alcohol by sex; simple effects tests showed that women woke up significantly

Table 3. Immediate and Residual Effects of Heavy Drinking on Measures of Subjective Ratings of Hangover and Neurocognitive Performance: Main Effects for Alcohol Versus Placebo and Significant Interactions

\begin{tabular}{|c|c|c|c|c|c|}
\hline Measure & After alcohol $M(\mathrm{SD})$ & After placebo $M(\mathrm{SD})$ & $F$ & df & $f^{\mathrm{a}}$ \\
\hline \multicolumn{6}{|l|}{ At peak BrAC } \\
\hline Ability to drive vehicle ${ }^{b}$ & $1.52(0.58)$ & $2.86(0.35)$ & $390.66^{\star * *}$ & 1,91 & 2.00 \\
\hline Likely to drive vehicle ${ }^{c}$ & $1.55(0.95)$ & $4.09(1.09)$ & $366.68^{\star \star *}$ & 1,92 & 2.00 \\
\hline \multicolumn{6}{|l|}{ Next morning } \\
\hline Ability to drive vehicle $\mathrm{b}^{\mathrm{b}}$ & $3.60(0.55)$ & $3.12(0.51)$ & $51.89^{\star \star \star}$ & 1,91 & 0.75 \\
\hline Likely to drive vehicle ${ }^{c}$ & $3.46(1.22)$ & $4.25(0.88)$ & $49.72^{\star \star \star}$ & 1,92 & 0.73 \\
\hline Acute Hangover Scale & $1.60(0.90)$ & $0.60(0.46)$ & $130.19^{\star \star \star}$ & 1,91 & 1.20 \\
\hline Vodka condition & $1.38(0.74)$ & $0.57(0.32)$ & $5.69^{d *}$ & 1,91 & $0.25^{d}$ \\
\hline Bourbon condition & $1.88(1.01)$ & $0.64(0.59)$ & & & \\
\hline Stanford Sleepiness Scale (SSS) ${ }^{\mathrm{e}}$ & 3.37 (1.22) & $2.61(1.12)$ & $29.81^{* * *}$ & 1,92 & 0.57 \\
\hline Sleep quality mean score ${ }^{f}$ & $2.98(0.64)$ & $3.46(0.53)$ & $45.97^{\star \star \star}$ & 1,88 & 0.72 \\
\hline
\end{tabular}

${ }^{*} p<0.05,{ }^{* \star} p<0.01$, and $p<0.001$.

affect size: $<0.25$ is small, 0.25 to 0.39 is medium, $>0.40$ is large.

"Rated from 1 "much worse" to 5 "much better."

"Rated from 1 "definitely would not" to 5 "definitely would."

dTest of interaction of alcohol and congener content.

eHigh score (7) is extremely sleepy.

fHigh score is "much better" or "excellent." 
Table 4. Residual Effects of Heavy Drinking on Measures of Neurocognitive Performance and Sleep Recordings: Main Effects for Alcohol Versus Placebo and Significant Interactions

\begin{tabular}{|c|c|c|c|c|c|}
\hline Measure & After alcohol $M(\mathrm{SD})$ & After placebo $M(\mathrm{SD})$ & $F$ & df & $f^{\mathrm{a}}$ \\
\hline \multicolumn{6}{|c|}{ Tests requiring sustained attention and/or reaction time } \\
\hline CPT reaction time (millisecond) & $387(42)$ & $379(38)$ & $10.28^{\star \star}$ & 1,90 & 0.33 \\
\hline PVT (millisecond) & $229.8(30.9)$ & $220.8(24.5)$ & $19.14^{\star \star \star}$ & 1,93 & 0.45 \\
\hline Finger Tapping Test: preferred hand & $57.1(6.7)$ & $58.0(6.5)$ & $4.45^{\ddagger}$ & 1,87 & 0.23 \\
\hline Alcohol on first night & $57.8(6.3)$ & $57.0(6.3)$ & $14.43^{\mathrm{b} * \star *}$ & 1,87 & $0.40^{\mathrm{b}}$ \\
\hline Alcohol on second night & $56.6(7.0)$ & $58.8(6.6)$ & & & \\
\hline \multicolumn{6}{|c|}{ Polysomnography variables hypothesized to be disrupted } \\
\hline Sleep efficiency ${ }^{\mathrm{C}}(\%$ of time asleep) & $90.1(7.4)$ & $92.2(5.5)$ & $12.10^{\star \star \star}$ & 1,83 & 0.37 \\
\hline Percent of time in REM sleep & $20.3(6.3)$ & $24.0(6.9)$ & $24.96^{\star \star \star}$ & 1,83 & 0.55 \\
\hline Wake time after sleep onset (minutes) & $33.4(24.7)$ & $25.1(18.8)$ & $6.66^{\star \star}$ & 1,83 & 0.28 \\
\hline \multicolumn{6}{|l|}{ Number of awakenings } \\
\hline Men & $13.4(6.3)$ & $15.0(6.3)$ & $7.21^{d_{*}}$ & 1,83 & $0.30^{d}$ \\
\hline Women & $13.2^{\mathrm{e}}(5.3)$ & $11.7^{\mathrm{e}}(6.3)$ & & & \\
\hline \multicolumn{6}{|l|}{ Other polysomnography variables } \\
\hline Percent of time in Slow Wave sleep & $26.6(7.7)$ & $24.7(6.9)$ & $6.90^{\star *}$ & 1,83 & 0.29 \\
\hline
\end{tabular}

${ }^{*} p<0.01,{ }^{* *} p<0.002,{ }^{* * *} p<0.001,{ }^{\star} p<0.05$ (trend).

aEffect size: $<0.25$ is small, 0.25 to 0.39 is medium, $>0.40$ is large.

${ }^{\mathrm{b}}$ Statistics for interaction of alcohol with order of administration.

${ }^{c}$ Variable transformed to correct skewness; raw variables presented to ease interpretation.

${ }^{d}$ Statistics for interaction of alcohol with gender.

'Values significantly different from each other in MANOVA simple effects tests, $p<0.05$.

CPT, Continuous Performance Test; PVT, Psychomotor Vigilance Task; REM, rapid eye movement.

$(p<0.05)$ more times after alcohol than placebo, $F(1,83)=4.74$, nearly medium effect $f=0.24$, but men did not, $F(1,83)=2.95$. No other effects were significant among these variables.

As is common after heavy drinking, amount of time in slow wave sleep increased after alcohol compared to placebo, with a medium effect size. No interaction effects were significant. No effects were significant for total sleep time, percent time in Stage 1 or 2 sleep, or sleep onset latency. Total sleep time across conditions was $M=7.2( \pm 0.5)$ hours, and sleep onset took $8.4( \pm 10.1)$ minutes.

Sleep Quality Ratings and Sleepiness. Reliability of the 4item sleep quality scale was good (Cronbach's $\alpha=0.74$ after placebo, 0.77 after alcohol). The mean of the sleep quality ratings indicated subjectively poorer sleep after alcohol than placebo, with a large effect size (see Table 3). The SSS score was significantly higher after alcohol than placebo. No effects of order, sex, or congeners were found in any analyses.

\section{Subjective Ratings of Performance}

Reliability among the 3 items was checked for the morning and evening separately, and separately after alcohol versus placebo. The items did not form a reliable scale across these sets of analyses so needed to be analyzed separately. As the ability item and the drinking attribution item were collinear after alcohol $(r>0.80)$, the attribution about drinking affecting ability was not used. The ability item correlated with the likelihood of driving item only $r=0.49$ to 0.51 in the evening after drinking alcohol or after placebo $(r=-0.41$ to -0.53 in the morning) so provided reasonably separate information.
For evening rating of ability to drive, a main effect was found for alcohol, with no significant interactions with order, gender or congener. For evening rating of likelihood that they would drive a vehicle, a main effect of alcohol was found, again with an extremely large effect size, but significant interactions were also found with order, $F(1,92)=4.09$, $p<0.05$, small effect size $f=0.21$, and with gender, $F(1,92)=5.90, p<0.02$, smallest medium effect size $f=$ 0.25 . The difference between alcohol and placebo ratings was greater when alcohol was first $(M$ difference $=2.82)$ than when alcohol was second $(M$ difference $=1.28)$. There was essentially no difference in ratings by gender after placebo ( $M=4.03$ males, $M=4.12$ females) but a larger difference after alcohol ( $M=1.89$ males and $M=1.33$ females). In the morning, significant alcohol effects were found with large effect sizes for both ratings, with no significant interaction effects. People felt less impaired but also less likely to drive after alcohol than placebo.

\section{Hangover's Correlations With Disrupted Sleep and Neuropsychological Performance Measures}

As can be seen in Table 5, hangover (AHS) was rated as more intense among people who had less efficient sleep, were awake more, and spent less time in REM sleep, supporting the role of alcohol-induced sleep deficits in the experience of hangover. Subjective sleepiness (SSS) the next morning also was higher for those reporting higher hangover. All relationships showed a fairly small amount of shared variance, with only the relationship with sleep efficiency being even a medium statistical effect size. People who had higher AHS scores also had significantly poorer scores on the 2 neurocognitive measures that were affected by alcohol: CPT and PVT. 
Table 5. Correlations of Acute Hangover Scale With Measures of Neurocognitive Performance and Sleep That Were Impaired by Alcohol: Alcohol Condition Only

\begin{tabular}{lrrrr}
\hline Measure & $r^{\mathrm{a}}$ & $n$ & $p$ & $\%$ variance \\
\hline CPT reaction time (milliseconds) & 0.26 & 90 & 0.007 & 7 \\
Psychomotor Vigilance Task & 0.26 & 91 & 0.006 & 7 \\
$\quad$ (PVT; milliseconds) & & & & \\
Percent of time in REM sleep & -0.23 & 85 & 0.02 & 5 \\
Wake time after sleep onset (minutes) & 0.23 & 85 & 0.02 & 5 \\
Sleep efficiency (\% of time asleep) & -0.33 & 85 & 0.001 & 11 \\
Stanford Sleepiness Scale & 0.25 & 90 & 0.01 & 6 \\
\hline
\end{tabular}

${ }^{\star} p<0.01,{ }^{* \star} p<0.002,{ }^{\star \star \star} p<0.001$, and ${ }^{\star} p<0.05$ (trend).

${ }^{a}$ Effect size: absolute value of $r<0.30=$ small, 0.30 to $0.49=$ medium

bVariable transformed to correct skewness.

CPT, Continuous Performance Test.

\section{Mediating Variables}

The mediators of impaired performance that we chose to test were sleep efficiency and time in REM sleep (time awake is collinear with sleep efficiency so redundant). The predictor variables were the 2 with significant residual effects of alcohol: PVT time and CPT reaction time. Each of these 4 variables was averaged across the alcohol and placebo conditions for these analyses. None of the relationships of sleep to performance was significant in regressions $\left(R^{2}\right.$ from 0.000 to 0.027 , all $F \mathrm{~s}(1,83) \leq 2.19)$, so mediation was not supported.

\section{DISCUSSION}

Drinking to above $0.10 \mathrm{~g} \% \mathrm{BrAC}$ results in residual effects on ability to perform complex cognitive tasks the next morning after alcohol has left the body. While drinking did not have residual effects on tasks that involved either speed or sustained attention but not both, tasks that required speed in using sustained attention were significantly impaired the next morning with medium to large sized effects. As drinking to this level affects complex cognitive abilities the next morning, safety could be affected (Howland et al., 2006). Attentional processing (both sustained and selective) and reaction time along with decision making are tasks considered to be involved in safe automobile driving (Allen et al., 2009) and are likely involved in other safety-sensitive occupational tasks. For example, residual effects of alcohol have been found to impair aspects of ability to fly aircraft that require vigilance across tasks (Yesavage and Leirer, 1986; Yesavage et al., 1994) while not affecting ship engineers' ability to restart malfunctioning engines (Rohsenow et al., 2006). It is interesting that participants did not think their driving ability was impaired in the morning. Although they did say they would be less willing to drive the morning after alcohol than after placebo, this could be because of the hangover they reported feeling as they did not perceive themselves as impaired.

Beverage congeners in bourbon versus vodka did significantly increase the intensity of hangover that was felt, consistent with results from studies in the 1970s. However, these had no effect on next-day performance, sleep, or perceived impairment either acutely or the next morning, consistent with the only other study that investigated this question (Seppälä et al., 1976). Thus, as congener content affects only how people feel the next day, the congeners in bourbon versus vodka do not appear to increase risk.

Effects of this amount of alcohol on subjective and objective measures of sleep are largely consistent with other studies. After alcohol, sleep was disrupted, characterized by lower sleep efficiency because of spending more time awake during the night, and less time was spent in REM sleep. Participants also reported having slept less well the next morning and feeling sleepier (tiredness is a symptom of hangover, Rohsenow et al., 2007). While effects on sleep efficiency were not found by Roehrs and colleagues (1991), possibly because of a lower dose of alcohol, increased wakefulness is often found, and reduced time in REM sleep is consistent with many other studies. When participants did sleep, their sleep was deeper as indicated by more slow wave sleep, also found in other studies. While we did not find predicted effects on time in Stage 1 sleep, effects on this stage have been inconsistent across studies, as reviewed in the introduction. Thus, drinking impaired sleep in a number of ways. However, the effects of alcohol on sleep were not found to mediate the effects of alcohol on performance the next day. As the sleep disrupting effects of alcohol did not account for the impaired cognitive performance, other mechanisms of these residual effects need to be explored.

Impaired sleep did correlate significantly with hangover symptoms. Correlations with hangover were significant for sleep efficiency, amount of time awake during the night, and time in REM sleep, but the largest effect size was seen with sleep efficiency. People who spent less of their time actually sleeping while in bed felt worse the next morning. While subjective sleepiness also correlated with hangover, tiredness is a component of hangover so this represents overlap in constructs rather than a meaningful relationship.

Hangover symptoms might be a mechanism by which drinking to intoxication impairs performance the next morning. While a true mediation model could not be tested, drinking to this level did increase hangover, and higher hangover scores after alcohol did correlate significantly with poorer performance scores on the neurocognitive measures that had been affected by alcohol. Thus, hangover symptoms might be contributing to impaired performance.

The study had several limitations. First, only young adults were used, to maximize safety, and it is possible that effects would differ in older adults with longer drinking histories and possibly more behavioral tolerance. Second, we excluded people with probable alcohol dependence yet such people are more likely to drink to intoxication on a regular basis. Third, for safety reasons, the target $\mathrm{BrAC}$ was set to the minimum at which hangover is reliably induced. Higher doses would produce more hangover, probably more next-day impairment, and more sleep disruption. Fourth, because of the high dose, most people knew what beverage they received so the 
influence of expectancy effects cannot be ruled out. Placebos are typically not effective at BrACs $\geq 0.07 \mathrm{~g} \%$ but these beliefs are usually not checked (Rohsenow and Marlatt, 1981).

Despite these limitations, this study adds to the relatively sparse experimental literature on the nature of hangover and other residual effects of alcohol. In particular, information was added on the types of performance decrements affected residually by heavy drinking, information that could be useful for safety-sensitive occupations. Furthermore, the study contributes to our understanding about possible mechanisms by which drinking to intoxication may produce effects on hangovers or on performance decrements the next day.

\section{ACKNOWLEDGMENTS}

This research was supported by (i) the National Institute on Alcohol Abuse and Alcoholism (NIAAA) Grant \#R01 AA12087 to the Boston University School of Public Health; (ii) by the National Center for Research Resources, General Clinical Research Center Grant M01 RR00533; and (iii) the Youth Alcohol Prevention Center, Boston University School of Public Health, with funding from NIAAA Grant \#P60 AA013759-01.

\section{REFERENCES}

Alford C, Wadling S (2004) Comparative effects of caffeine and alcohol bedtime drinks on sleep, performance and mood in young adults. J Psychopharmacol 18(Suppl.):A41.

Allen AJ, Meda SA, Skudlarski P, Calhoun VD, Astur R, Ruopp KC, Pearlson GD (2009) Effects of alcohol on performance on a distraction task during simulated driving. Alcohol Clin Exp Res 33:617-625.

Ames G, Grube J, Moore R (1997) The relationship of drinking and hangovers to workplace problems: an empirical study. J Stud Alcohol 58:37-47.

Brick J, Nathan PE, Westrick E, Frankenstein W, Shapiro A (1986) The effects of menstrual cycle on blood alcohol levels and behavior. J Stud Alcohol 47:472-477.

Calder I (1997) Editorial: hangovers. Not the ethanol-perhaps the methanol. Br Med J 314:2-3.

Chait LD, Perry JL (1994) Acute and residual effects of alcohol and marijuana, alone and in combination, on mood and performance. Psychopharmacology 115:340-349.

Chapman L (1970) Experimental induction of hangover. Q J Stud Alcohol (Suppl. 5):67-86.

Cohen J (1988) Statistical Power Analysis for the Behavioral Sciences, 2nd ed. Lawrence Erlbaum, Mahwah, NJ.

Dar R, Serlin RC, Omer H (1994) Misuse of statistical tests in three decades of psychotherapy research. J Consult Clin Psychol 62:75-82.

Dinges DF, Powell JE (1985) Microcomputer analyses of performance on a portable, simple visual RT task during sustained operations. Behav Res Methods Instrum Comput 17:652-655.

Dowd PJ, Wolfe JW, Cramer RL (1973) Aftereffects of alcohol on the perception and control of pitch attitude during centripetal acceleration. Aerosp Med 44:928-930.

Finnigan F, Hammersley R, Cooper T (1998) An examination of next-day hangover effects after a $100 \mathrm{mg} / 100 \mathrm{ml}$ dose of alcohol in heavy social drinkers. Addiction 93:1829-1838.

Finnigan F, Schulze D, Smallwood J, Helander A (2005) The effects of selfadministered alcohol-induced "hangover" in a naturalistic setting on psychomotor and cognitive performance and subjective state. Addiction 100:1680-1689.
Friel PN, Logan BK, O’Malley D, Baer JS (1999) Development of dosing guidelines for reaching selected target breath alcohol concentrations. J Stud Alcohol 60:555-565.

Gillin JC, Drummond SPA, Clark CP, Moore P (2005) Medication and substance abuse, in Principles and Practice of Sleep Medicine, 4th ed (Kryger MH, Roth T, Dement WC eds), pp. 1345-1358. Elsevier Saunders, Philadelphia.

Hoddes E, Dement WC, Zarcone V (1973) Quantification of sleepiness: a new approach. Psychophysiology 10:431-436.

Howland J, Almeida A, Rohsenow D, Minsky S, Greece J (2006) How safe are federal regulations on occupational alcohol use? J Public Health Policy 27:389-404.

Jones AW (1987) Elimination half-life of methanol during hangover. Pharmacol Toxicol 60:217-220.

Katkin ES, Hayes WN, Teger AI, Preuitt DG (1970) Effects of alcoholic beverages differing in congener content on psychomotor tasks and risk-taking. Q J Stud Alcohol (Suppl. 5):101-114.

Kim DJ, Yoon SJ, Lee HP, Choi BM, Go HJ (2003) The effects of alcohol hangover on cognitive functions in healthy subjects. Int J Neurosci 113:581594.

Knowles JB, Laverty SG, Kuechler HA (1968) Effect of alcohol on REM sleep. Q J Stud Alcohol 29:342-349.

Kobayashi T, Misaki K, Nakagawa H, Okuda K, Ota T, Kanda I, Isaki K, Kosino Y, Fukuda H (1998) Alcohol effect on sleep electroencephalography by fast Fourier transformation. Psychiatry Clin Neurosci 52:154-155.

Kruisselbrink LD, Martin KL, Megeney M, Fowles JR, Murphy RJ (2006) Physical and psychomotor functioning of females the morning after consuming low to moderate quantities of beer. J Stud Alcohol 67:416-420.

Lemon J, Chesher G, Fox A, Greeley J, Nabke C (1993) Investigation of the "hangover" effects of an acute dose of alcohol on psychomotor performance. Alcohol Clin Exp Res 17:665-668.

MacKinnon DP, Lockwood CM, Hoffman JM, West SG, Sheets V (2002) A comparison of methods to test mediation and other intervening variable effects. Psychol Method 7:83-104.

MacLean AW, Cairns J (1982) Dose-response effects of ethanol on the sleep of young men. J Stud Alcohol 43:434 444.

Mangione T, Howland J, Amick B, Cote J, Lee M, Bell N, Levine S (1999) Employee drinking practices and work performance. J Stud Alcohol 60:261-270.

McCaul ME, Turkkan JS, Svikis DS, Bigelow GE (1991) Alcohol and secobarbital effects as a function of familial alcoholism: extended intoxication and increased withdrawal effects. Alcohol Clin Exp Res 15:94-101.

McKinney A, Coyle K (2004) Next day effects of a normal night's drinking on memory and psychomotor performance. Alcohol Alcohol 39:509-513.

McMurphree HB, Price LM, Greenberg LA (1966) Effect of congeners in alcoholic beverages on the incidence of nystagmus. Q J Stud Alcohol 27:201-213.

Morrow D, Leirer V, Yesavage J (1990) The influence of alcohol and aging on radio communication during flight. Aviat Space Environ Med 61:12-20.

Morrow D, Leirer V, Yesavage J, Tinklenberg J (1991) Alcohol, age, and piloting: judgment, mood, and actual performance. Int J Addict 26:669683.

Morrow D, Yesavage J, Leirer V, Dohlert N, Taylor J, Tinkleberg J (1993) The time-course of alcohol impairment of general aviation pilot performance in a Frasca 141 Simulator. Aviat Space Environ Med 64:697-705.

Myrsten AL, Ryberg U, Idestrom CM, Lamble R (1980) Alcohol intoxication and hangover: modification of hangover by chlormethiazole. Psychopharmacology 69:117-125.

Nathan PE, Zare NC, Ferneau EW, Jr, Lowenstein LM (1970) Effects of congener differences in alcoholic beverages on the behavior of alcoholics. Q $\mathrm{J}$ Stud Alcohol 5 (Suppl. 5):87-100.

Niaura RS, Nathan PE, Frankenstein W, Shapiro AP, Brick J (1987) Gender differences in acute psychomotor, cognitive, and pharmacokinetic response to alcohol. Addict Behav 12:345-356.

Prat G, Adan A, Perez-Paimes M, Sanchez-Turet M (2008) Neurocognitve effects of alcohol hangover. Addict Behav 33:15-23. 
Roehrs T, Roth T (2001a) Sleep, sleepiness, and alcohol use. Alcohol Res Health 25:101-109.

Roehrs T, Roth T (2001b) Sleep, sleepiness, sleep disorders and alcohol use and abuse. Sleep Med Rev 5:287-297.

Roehrs T, Yoon J, Roth T (1991) Nocturnal and next-day effects of ethanol and basal level of sleepiness. Hum Psychopharmacol 6:307-311.

Rohsenow DJ, Howland J, Minsky S, Arnedt JT (2006) Effects of heavy drinking by maritime academy cadets on hangover, perceived sleep, and next day ship power plant operation. J Stud Alcohol 67:405-415.

Rohsenow DJ, Howland J, Minsky SJ, Greece J, Almeida A, Roehrs T (2007) The Acute Hangover Scale: a new measure of immediate hangover symptoms. Addict Behav 32:1314-1320.

Rohsenow DJ, Marlatt GA (1981) The balanced placebo design: methodological considerations. Addict Behav 6:107-122.

Rundell OH, Lester BK, Griffiths WS, Williams HL (1972) Alcohol and sleep in young adults. Psychopharmacologia 26:201-218.

Selzer MD, Vinokur A, Van Rooijen L (1975) A self-administered Short Michigan Alcoholism Screening Test (SMAST). J Stud Alcohol 36:117126.

Seppälä T, Leino T, Linnoila M, Huttunen M, Ylikahri R (1976) Effects of hangover on psychomotor skills related to driving: modification by fructose and glucose. Acta Pharmacol Toxicol 38:209-218.

Singleton RA Jr, Wolfson AR (2009) Alcohol consumption, sleep, and academic performance among college students. J Stud Alcohol Drugs 70:355363.

Stone BM (1980) Sleep and low doses of alcohol. Electroencephalogr Clin Neurophysiol 4:706-709.

Streufert S, Pogash R, Braig D, Gingrich D, Kantner A, Landis R, Lonardi L, Roache J, Severs W (1995) Alcohol hangover and managerial effectiveness. Alcohol Clin Exp Res 19:1141-1146.

Swift R, Davidson D (1998) Alcohol hangover: mechanisms and mediators. Alcohol Health Res World 22:54-60.

Tabachnick BG, Fidell LS (1996) Using Multivariate Statistics, 3rd ed. Harper Collins, New York.

Tassi P, Muzet A (2000) Sleep inertia. Sleep Med Rev 4:341-353.
Taylor J, Dohlert N, Morrow D, Friedman L, Yesavage J (1994) Acute and 8hour effects of alcohol $(0.08 \%$ BAC) in younger and older pilots' simulator performance. Aviat Space Environ Med 65:718-725.

Taylor JL, Dolhert N, Friedman L, Mumenthaler M, Yesavage JA (1996) Alcohol elimination and simulator performance of male and female aviators: a preliminary report. Aviat Space Environ Med 67:407-413.

Terner JM, deWit H (2006) Menstrual cycle phase and responses to drugs of abuse in humans. Drug Alcohol Depend 84:1-13.

Törnros J, Laurell H (1991) Acute and hang-over effects of alcohol on simulated driving performance. Blutalkohol 28:24-30.

Verster JC, van Duin D, Volkerts ER, Schreude AH, Verbaten MN (2003) Alcohol hangover effects on memory functioning and vigilance performance after an evening of binge drinking. Neuropsychopharmacology 28:740-746.

White RF, James KE, Vasterling JJ, Letz RE, Marans K, Delaney R, Krengel M, Rose F, Kraemer HC (2003) Neuropsychological screening for cognitive impairment using computer-assisted tasks. Assessment 10:86-101.

Wiese JG, Shlipak MG, Browner WS (2000) The alcohol hangover. Ann Int Med 132:897-902.

Williams DL, MacLean AW, Cairns J (1983) Dose-response effects of ethanol on the sleep of young women. J Stud Alcohol 44:515-523.

Wolkenberg R, Gold C, Tichauer E (1975) Delayed effects of acute alcoholic intoxication on performance with reference to work safety. J Safety Res 7:104-119.

Yesavage J, Dolhert N, Taylor J (1994) Flight simulator performance of younger and older aircraft pilots: effects of age and alcohol. J Am Soc Geriatr Dent 42:577-582.

Yesavage J, Leirer V (1986) Hangover effects on aircraft pilots 14 hours after alcohol ingestion: a preliminary report. Am J Psychiatry 143:1546-1550.

Ylikahri RH, Huttumen MO, Eriksson CJP, Nikkilä EA (1974) Metabolic studies on the pathogenesis of hangover. Eur J Clin Invest 4:93-100.

Yules RB, Freedman DX, Chandler KA (1966) The effect of ethyl alcohol on man's electroencephalographic sleep cycle. Electroencephalogr Clin Neurophysiol 20:109-111.

Yules RB, Lippman ME, Freedman DX (1967) Alcohol administration prior to sleep: the effect on EEG sleep stages. Arch Gen Psychiatry 19:94-97. 